\title{
COVID-19 Screening for Hospitalized Patients: The Role of Expanded Hospital Surveillance in a Low Prevalence Setting
}

\author{
Jen-Yu Hsu iD ' \\ Po-Yu Liu iD \\ Chien-Hao Tseng (iD) \\ Chia-Wei Liu' \\ Wan-Ting Yang (D) \\ Wei-Hsuan Huang' \\ Shu-Yuan Li (D) ' \\ Ya-Chun Liao (iD) \\ Ming-Ju Wu (iD ${ }^{2}$ \\ 'Division of Infectious Diseases, \\ Department of Internal Medicine, \\ Taichung Veterans General Hospital, \\ Taichung, 407, Taiwan; ${ }^{2}$ Division of \\ Nephrology, Department of Internal \\ Medicine, Taichung Veterans General \\ Hospital, Taichung 407, Taiwan \& College \\ of Medicine, National Chung Hsing \\ University, Taichung, 402, Taiwan
}

Correspondence: Ming-Ju Wu Department of Internal Medicine, Taichung Veterans General Hospital, No. 1650, Sec. 4, Taiwan Blvd, Xitun Dist, Taichung City, 407219, Taiwan Tel +886-4-23592525

Emailwmj530@gmail.com
Purpose: The COVID-19 pandemic poses a serious threat to healthcare workers and hospitalized patients. Early detection of COVID-19 cases is essential to control the spread in healthcare facilities. However, real-world data on the screening criteria for hospitalized patients remain scarce. We aimed to explore whether patients with negative results of prehospital screening for COVID-19 should be rescreened after admission in a low-prevalence (less than $3 \%$ of the world average) setting.

Patients and Methods: We retrospectively included patients in central Taiwan who were negative at the first screening but were newly diagnosed with pneumonia or had a body temperature above 38 degrees Celsius during their hospitalization. Each patient might be included as an eligible case several times, and the proportions of cases who were rescreened for COVID-19 and those diagnosed with COVID-19 were calculated. A logistic regression model was constructed to identify factors associated with rescreening. Reverse transcriptionpolymerase chain reaction tests were used to confirm the diagnosis of COVID-19.

Results: A total of 3549 cases eligible for COVID-19 rescreening were included. There were 242 cases $(6.8 \%)$ who received rescreening. In the multivariable analysis, cases aged 75 years or older, those with potential exposure to SARS-CoV-2, or patients visiting specific departments, such as the Cardiovascular Center and Department of Neurology, were more likely to be rescreened. None was diagnosed with COVID-19 after rescreening. There was no known cluster infection outbreak in the hospital or in the local community during the study period and in the following two months.

Conclusion: In Taiwan, a country with a low COVID-19 prevalence, it was deemed safe to rescreen only high-risk hospitalized patients. This strategy was effective and reduced unnecessary costs.

Keywords: fever, pneumonia, risk, SARS-CoV-2, screen

\section{Introduction}

As of September 20, 2021, the total number of confirmed cases of coronavirus disease 2019 (COVID-19), a disease caused by the Severe Acute Respiratory Syndrome coronavirus 2 (SARS-CoV-2), reached 228.4 million, which includes 4.7 million deaths worldwide. ${ }^{1}$ In contrast, Taiwan has had relatively few confirmed and fatal cases, at 16,147 and 840, respectively. COVID-19 is highly contagious and is mainly transmitted through respiratory droplets and close contact with an infected individual. In addition to causing community spread, COVID-19 accounts for a high proportion of nosocomial infections, and thus poses a huge threat to healthcare workers and 
hospitalized patients. ${ }^{2}$ The spread of COVID-19 in healthcare facilities may lead to shortages of both medical personnel and supplies, and hence preventive actions should be taken as soon as possible. ${ }^{3}$

The main clinical characteristics of COVID-19 patients vary greatly. The leading clinical symptoms and signs are fever, fatigue, headache, olfactory or gustatory dysfunction, nasal obstruction, rhinorrhea, cough, sore throat, gastrointestinal symptoms, lymphopenia, prolonged prothrombin time, and elevated inflammatory markers, such as C-reactive protein, procalcitonin, erythrocyte sedimentation rate, lactate dehydrogenase, and d-dimer, ${ }^{4-6}$ while dyspnea, leukocytosis, increased inflammatory markers, and elevation of creatinine and aspartate aminotransferase indicate greater disease severity. ${ }^{7,8}$ The predictors of SARS-CoV-2 infection include contact history with confirmed cases, fever, respiratory symptoms, gustatory dysfunction, gastrointestinal symptoms, and neurological symptoms. ${ }^{9-12}$ The results of laboratory examinations have also been studied to help construct a predictive model using artificial intelligence. ${ }^{13}$ However, patients with early COVID-19 or certain groups of patients, such as pregnant women, may only experience mild symptoms or could even be asymptomatic. ${ }^{14,15}$ Chest computed tomography, which often shows bilateral patchy shadows or ground glass opacity over the peripheral lungs in COVID-19 patients, is a sensitive tool that can be helpful in the diagnosis of COVID19 when the clinical presentation and molecular test results are conflicting. ${ }^{5,6,8,16,17}$ However, it is not routinely used as a screening tool. Isolation of high-risk patients is not enough to prevent nosocomial infections, and therefore universal screening for COVID-19 using a Reverse TranscriptionPolymerase Chain Reaction (RT-PCR) test to detect SARSCoV-2 remains necessary prior to hospitalization. ${ }^{15,18}$ Nevertheless, it is important to note that negative results from screening at admission may not be used as a basis for estimating the risks of nosocomial transmission. ${ }^{19}$

Nosocomial infections due to COVID-19 are not easy to prevent, and it remains difficult to immediately control the spread without proper intervention measures. Several studies have suggested that routine monitoring of COVID19 transmission should be carried out for high-risk groups, such as patients receiving hemodialysis ${ }^{20,21}$ and patients hospitalized for hemato-oncological diseases. ${ }^{22-24}$ Other vulnerable patients, such as those in the intensive care units, may also require special attention. ${ }^{25}$ Universal COVID-19 screening for all hospitalized patients is a resource-intensive task, but it could sometimes still play a key role in limiting the spread from the community, given the inseparable connection between healthcare institutions and their neighboring communities. ${ }^{26}$

Although the results of COVID-19 screening prior to hospitalization vary with its prevalence, ${ }^{27-32}$ pre-hospital screening has been widely recommended. ${ }^{27,28,30-32}$ However, real-world data on the screening criteria for hospitalized patients remain scarce. In this study we aimed to explore whether patients with negative results of pre-hospital screening for COVID-19 who develop fever or pneumonia after admission should be rescreened in a low-prevalence setting.

\section{Materials and Methods Study Population}

This study was conducted at Taichung Veterans General Hospital, a medical center with a 1600-bed capacity in central Taiwan. In response to COVID-19 cluster infection outbreaks, the Taiwan government raised the COVID-19 alert to Level 3 on May 19, 2021, restricting both indoor and outdoor gatherings. The alert was then reduced to Level 2 on July 26, 2021. All hospitalized patients at Taichung Veterans General Hospital received their first screening for COVID-19 between May 15, 2021 and May 17, 2021. All patients who were admitted after May 18, 2021 received their first screening for COVID-19 before being hospitalized. We included patients who were negative at the first screening but were newly diagnosed with pneumonia or had a body temperature above 38 degrees Celsius during their hospitalization between May 18, 2021 and August 9, 2021. Those who were positive for screening or rescreening were transferred to a negative-pressure isolation room and were subsequently excluded from the study.

\section{Data Collection}

A standardized case record form was used to collect information on gender, age, history of travel, occupation, contacts, and cluster activity, as well as the results of laboratory tests and image studies. Patients were assigned to 1 of 3 different quarantine levels: not quarantined for COVID-19, quarantined due to potential exposure to SARS-CoV-2, and quarantined due to unexplained community-acquired pneumonia. Patients belonged to different departments, including the Department of Internal Medicine, Surgery, Cardiovascular Center, Neurology, Pediatrics, Obstetrics and Gynecology, and others. If a hospitalized patient was newly diagnosed with pneumonia or had experienced a body temperature above 38 degrees 
Celsius within the previous 24 hours, the computer system would issue an automated warning reminding the clinician to consider the possibility that the patient was infected with COVID-19. A patient might be included as an eligible case several times if he or she met the inclusion criteria more than once. The decision to rescreen for COVID-19 was made at the discretion of the treating physicians.

\section{Case Definition of COVID-19}

To improve the efficiency in clinical practice, all cases were screened or rescreened by detecting SARS-CoV-2 with a nucleic acid amplification test using clinical specimens, such as nasopharyngeal or throat swabs, sputum, or lower respiratory tract aspirate. A COVID-19 case was defined as a patient with a positive SARS-CoV-2 result.

\section{Reverse Transcription-Polymerase Chain Reaction (RT-PCR) Assay}

In our study, the nucleic acid amplification test that was used to detect SARS-CoV-2 was the RT-PCR test. All clinical specimens were refrigerated at $4{ }^{\circ} \mathrm{C}$ and loaded onto a cobas ${ }^{\circledR}$ 6800 instrument (Roche Molecular Systems, Rotkreuz, Switzerland) within 24 hours. The cobas ${ }^{\circledR}$ SARS-CoV-2 test kits were used. The automated system targeted the SARS-CoV-2 envelope and nucleocapsid gene. The limit of detection was 100 copies per reaction (AccuPlex ${ }^{\mathrm{TM}}$ SARS$\mathrm{CoV}-2$ reference material kit). All protocols were performed according to the manufacturer's instructions.

\section{Statistical Analysis}

The proportions of cases who received rescreening for COVID-19 and those who were diagnosed with COVID-19 were calculated as the total number of occurrences divided by the total number of eligible cases, respectively. As for the associated variables, the median and interquartile range were calculated for quantitative variables, while percentages were calculated for categorical variables. The KolmogorovSmirnov test was used to evaluate the normality of variables. The Mann-Whitney $U$-test was used to compare quantitative variables, and the $\chi 2$ or two-tailed Fisher's exact test was used to compare categorical variables. A logistic regression model was constructed to identify any associated factors. Variables with a $P$ value $\leq 0.25$ in the univariable analysis were added in a stepwise manner, with only significant variables being used in the final, multivariable analysis. Gender and age were included in the final model regardless of the level of significance. All statistical analyses were conducted using Statistical
Analysis System software, version 9.4 (SAS Institute, Cary, NC, USA). A two-sided $P$ value $\leq 0.05$ was considered statistically significant.

\section{Ethics Statement}

This study was approved by the Clinical Ethics Committee of Taichung Veterans General Hospital (no. CE21384B). This study was conducted in accordance with the Declaration of Helsinki. The patients were informed about the objective and the data collection methods of the study, participated benefited potential risk of the study. Informed written consents were received prior to starting the study. The patients understood that they had the right to withdraw anytime if they wanted. All information was kept confidential without identification or name tag on it.

\section{Results}

A total of 3549 cases (1628 patients) eligible for COVID19 rescreening were included, with a median of one case per patient (interquartile range, 1-2). There were 242 rescreened cases $(6.8 \%)$; most of the patients were rescreened only once, and only 7 patients were rescreened twice. No patients were diagnosed with COVID-19 after rescreening. In addition, there was no known cluster infection outbreak in the hospital or in the local community during the study period and in the following two months.

The clinical characteristics of the rescreened and nonrescreened groups are shown in Table 1. No notable difference in gender proportion existed between the two groups, but the rescreened group had more people aged 75 years or older compared with the non-rescreened group (89/242, 36.8\% vs 690/3307, 20.9\%) ( $\mathrm{P}<0.0001)$. The median length of hospital stay was 4 days without a significant difference between the two groups. However, there were significant differences between the two groups regarding the quarantine levels of cases and the departments they belonged to.

Regarding the quarantine levels, 19.4\% (47/242) of rescreened cases had been quarantined due to potential exposure to SARS-CoV-2, which was much higher than the $2.1 \%(68 / 3307)$ of non-rescreened cases $(\mathrm{P}<0.0001)$. With respect to departments, rescreening was more frequently associated with the Department of Internal Medicine $(145 / 242,59.9 \%$ vs $1877 / 3307,56.8 \%)$, Cardiovascular Center (12/242, 5.0\% vs 56/3307, 1.7\%), Neurology $(35 / 242,14.5 \%$ vs $135 / 3307,4.1 \%)$, and other independent departments (19/242, 7.9\% vs 238/3307, $7.2 \%$ ), but was less frequently associated with the 
Table I Clinical Characteristics of Rescreened and Non-Rescreened Cases

\begin{tabular}{|c|c|c|c|}
\hline Variable $^{\mathbf{a}}$ & Rescreened & Non-Rescreened & \\
\hline Total Number & $N=242$ & $\mathbf{N}=3307$ & $P$ value ${ }^{b}$ \\
\hline \multicolumn{4}{|l|}{ Demographics } \\
\hline Male & $136(56.2)$ & 1870 (56.5) & 0.9160 \\
\hline Female & $106(43.8)$ & | 437 (43.5) & 0.9160 \\
\hline Age (years) & $66.7(51.0-80.3)$ & $61.3(48.3-72.8)$ & $<0.0001$ \\
\hline Age $\geq 75$ years & $89(36.8)$ & $690(20.9)$ & $<0.000$ I \\
\hline Hospitalization (days) & $4.0(1.0-10.0)$ & $4.0(2.0-11.0)$ & 0.0769 \\
\hline Different quarantine levels & & & $<0.0001$ \\
\hline Not quarantined for COVID-19 & $187(77.3)$ & $3147(95.2)$ & \\
\hline Quarantined due to potential exposure to SARS-CoV-2 & $47(19.4)$ & $68(2.1)$ & \\
\hline Quarantined due to unexplained community-acquired pneumonia & $8(3.3)$ & $92(2.8)$ & \\
\hline Departments & & & $<0.0001$ \\
\hline Internal Medicine & $145(59.9)$ & $1877(56.8)$ & \\
\hline Surgery & $22(9.1)$ & $616(18.6)$ & \\
\hline Cardiovascular Center & $12(5.0)$ & $56(1.7)$ & \\
\hline Neurology & $35(14.5)$ & $135(4.1)$ & \\
\hline Pediatrics & $5(2.1)$ & $208(6.3)$ & \\
\hline Obstetrics and Gynecology & $4(1.7)$ & $177(5.4)$ & \\
\hline Others & $19(7.9)$ & $238(7.2)$ & \\
\hline
\end{tabular}

Notes: ${ }^{a}$ Data are presented as median values (interquartile range) for continuous variables and number of cases (percentage) for categorical variables. ${ }^{b} P$ value: The MannWhitney U-test was used to compare continuous variables, and the $\chi^{2}$ or two-tailed Fisher's exact test was used to compare categorical variables.

Department of Surgery $(22 / 242,9.1 \%$ vs $616 / 3307$, $18.6 \%$ ), Pediatrics (5/242, 2.1\% vs 208/3307, 6.3\%), and Obstetrics and Gynecology (4/242, $1.7 \%$ vs 177/3307, $5.4 \%$ ), when compared with non-rescreening $(\mathrm{P}<0.0001)$. Within the Department of Internal Medicine, cases belonging to the Division of Infectious Diseases were more likely to be rescreened $(55 / 145$, $37.9 \%$ vs $286 / 1877,15.2 \%$ ), while cases belonging to the Division of Hematology and Oncology had a lower prevalence rate of rescreening $(14 / 145,9.7 \%$ vs $711 / 1877$, $37.9 \%)(\mathrm{P}<0.0001)$ (Table S1).

In the multivariable logistic regression model, the variables associated with rescreening included age, quarantine levels, and departments. Cases with an age of 75 years or older were more likely to be rescreened compared with younger cases (adjusted Odds Ratio [aOR] 1.56, 95\% Confidence Interval [CI] 1.16-2.10). Those who had been quarantined due to potential exposure to SARSCoV-2 were more likely to be rescreened compared with those receiving general care (aOR 11.63, 95\% CI 7.8017.35). Compared with cases in the Department of Internal Medicine, those attending the Cardiovascular Center (aOR 3.97, 95\% CI 2.05-7.69) or Department of Neurology
(aOR 4.69, 95\% CI 3.05-7.20) were more likely to be rescreened (Table 2).

\section{Discussion}

In this real-world experience of COVID-19 rescreening in a low-prevalence setting, we found that the proportion of cases being considered by clinicians to be rescreened because of pneumonia or fever was low, with the results of all rescreened cases coming back negative. Those who were elderly, had potential exposure to SARS-CoV-2, or were treated in a specific department, such as the Cardiovascular Center or Department of Neurology, were more likely to be rescreened. These findings may be of value to frontline medical personnel.

In Taiwan, an outbreak of COVID-19 cluster infections began in mid-May 2021, but the prevalence rate in midAugust 2021 was only 675 cases per million population, which was much lower than the global rate of 26,137 cases per million population. ${ }^{1}$ In addition, the dominant SARSCoV-2 strain in Taiwan was mainly the Alpha variant (B.1.1.7), which is known to be much less contagious than the Delta variant (B.1.617.2), resulting in a relatively slow spread of COVID-19. At the same time, a number of measures have been taken to prevent widespread exposure 
Table 2 Univariable and Multivariable Logistic Regression Analysis of Factors Associated with Rescreened Cases

\begin{tabular}{|c|c|c|c|c|}
\hline \multirow[t]{2}{*}{ Variable } & \multicolumn{2}{|c|}{ Univariable Analysis } & \multicolumn{2}{|c|}{ Multivariable Analysis } \\
\hline & Crude OR $(95 \% \mathrm{Cl})$ & $P$ value & Adjusted OR (95\% Cl) & $P$ value \\
\hline \multicolumn{5}{|l|}{ Gender } \\
\hline Male & I.00 (reference) & & I.00 (reference) & \\
\hline Female & $1.01(0.78-1.32)$ & 0.9159 & $1.17(0.88-1.55)$ & 0.2839 \\
\hline Age $\geq 75$ years & $2.21(1.68-2.90)$ & $<0.0001$ & $1.56(1.16-2.10)$ & 0.0035 \\
\hline Hospitalization (days) & $1.00(0.99-1.00)$ & 0.5222 & & \\
\hline \multicolumn{5}{|l|}{ Different quarantine levels } \\
\hline Not quarantined for COVID-19 & 1.00 (reference) & & 1.00 (reference) & \\
\hline Quarantined due to potential exposure to SARS-CoV-2 & $11.63(7.80-17.35)$ & $<0.0001$ & $11.76(7.64-18.10)$ & $<0.0001$ \\
\hline Quarantined due to unexplained community-acquired pneumonia & $1.46(0.70-3.06)$ & 0.3115 & $1.66(0.78-3.53)$ & 0.1881 \\
\hline \multicolumn{5}{|l|}{ Departments } \\
\hline Internal Medicine & I.00 (reference) & & 1.00 (reference) & \\
\hline Surgery & $0.46(0.29-0.73)$ & 0.0010 & $0.66(0.4 \mathrm{I}-1.07)$ & 0.0911 \\
\hline Cardiovascular Center & $2.77(1.45-5.29)$ & 0.0020 & $3.97(2.05-7.69)$ & $<0.0001$ \\
\hline Neurology & $3.36(2.23-5.05)$ & $<0.0001$ & $4.69(3.05-7.20)$ & $<0.0001$ \\
\hline Pediatrics & $0.31(0.13-0.77)$ & 0.0113 & $0.48(0.19-1.21)$ & 0.1221 \\
\hline Obstetrics and Gynecology & $0.29(0.11-0.80)$ & 0.0166 & $0.44(0.16-1.23)$ & 0.1180 \\
\hline Other & $1.03(0.63-1.70)$ & 0.8968 & $1.50(0.90-2.50)$ & 0.1218 \\
\hline
\end{tabular}

Abbreviations: $\mathrm{Cl}$, confidence interval; OR, odds ratio.

to SARS-CoV-2, including following hand hygiene protocols, wearing of protective face masks, disinfection of high-touch surfaces, maintaining strict adherence to social distancing, promoting telecommuting for work, preventing unnecessary activities, instituting enhanced quarantine measures, and enacting rigorous contact tracing. ${ }^{33,34}$ During the three-month period of rising infections, the coverage rate of at least one dose of vaccine being administered increased from $1 \%$ to $40 \%$, of which AstraZeneca accounted for $64 \%$ and Moderna accounted for $36 \%$. This proactive response also helped to prevent cluster infections. $^{35}$ In Taiwan, there was no large-scale nosocomial transmission or outbreaks of COVID-19, which was very different from the infection rates of up to $60 \%$ that were seen in other countries. ${ }^{36}$ In this study, in a lowprevalence setting, we found that for patients who were negative at the first screening prior to hospitalization, the subsequent rescreening of hospitalized high-risk individuals was sufficient to avoid nosocomial infections.

In our study, patients older than 75 years and those with potential exposure to SARS-CoV-2 were more likely to be rescreened, while the length of hospital stay was not related to the need for rescreening. Elderly people were determined to be more susceptible to COVID-19 infection, had poorer viral clearance, and exhibited a higher case fatality rate. Moreover, there were concerns surrounding cluster infections in long-term care facilities and therefore this target group was given additional attention. ${ }^{37}$ For people diagnosed with COVID-19, SARS-CoV-2 can be detected in the respiratory tract 2-3 days prior to the onset of symptoms, and it can be spread to contacts in as little as 15 minutes through airborne transmission. ${ }^{35}$ Those who have had potential exposure to SARS-CoV-2 and have not been vaccinated were apparently the highest risk group. The median time for rescreening in our study was 4 days, which is consistent with the possible latent infection period range of 1 to 5 days before the onset of disease. ${ }^{38}$

Another factor in our study associated with rescreening was the department that the patient attended. Compared with the Department of Internal Medicine where there were more patients with pneumonia or fever, cases in the Department of Neurology and those from the Cardiovascular Center were more likely to be rescreened. Although it has been recommended that emergency neurosurgery patients should be given priority and that elective neurosurgeries should be postponed during the COVID-19 epidemic, ${ }^{39,40}$ it should be borne in mind that any lack or delay of appropriate intervention may lead to deterioration of neurological function. ${ }^{40,41}$ 
Therefore, for patients whose neurosurgery should not be delayed, mandatory preoperative screening for COVID-19 was proposed to help minimize the risks, as stipulated in the clinical practice guidelines. ${ }^{42}$ As with neurosurgery, scheduled invasive interventions were reserved for emergent cardiac patients. $^{43-46}$ Additionally, multiple reports have suggested that patients with underlying cardiovascular comorbidities such as heart failure are at higher risk of severe disease and mortality due to COVID-19. ${ }^{44,47-50}$ Collectively, the rescreening of patients with worsening cardiovascular disease was indeed necessary. For patients attending the Department of Internal Medicine, the higher rescreening rate in the Division of Infectious Diseases may be because approximately half of the quarantined patients belonged to that division, while the lower rescreening rate in the Division of Hematology and Oncology could possibly be explained by the fact that around three-quarters of the cases were repeatedly included due to recurrent fever caused by the underlying disease or anticancer therapy.

Our study had several limitations. First, this study was conducted in a medical center with a low prevalence of COVID-19. The results reported herein may not be generalizable to other institutions with a higher prevalence of COVID-19. Hospitals in areas with moderate to high COVID-19 prevalence or a dominant SARSCoV-2 Delta variant may require more expanded surveillance. More symptoms or signs should be included in the rescreening criteria or hospitalized patients should be extensively rescreened. Second, because this was a retrospective study and most information could only be retrieved from medical records, we were unable to clarify the condition of the disease in patients and the reasons for rescreening or not rescreening patients. Vaccination was an important factor that may affect the decision to rescreen, but complete information could not be obtained in this study because people generally received vaccination outside of hospitals in Taiwan. Nevertheless, we attempted to determine highrisk groups based upon quarantine levels and the departments in which they were being treated. Third, the heterogeneity of patients was large. Some patients who had persistent fever due to another disease would be repeatedly included. Moreover, the rescreening strategies in different departments varied greatly, and thus it was difficult to formulate rules. More research is needed to explore surveillance of COVID-19 in a hospital setting.

\section{Conclusion}

The proportion of rescreened cases was low, and the results of all rescreened cases came back negative. There was also no known subsequent cluster infection outbreak. This study determined that in a hospital with a low COVID-19 prevalence, it was safe to rescreen only highrisk cases, such as the elderly, those with potential prior exposure to SARS-CoV-2, and patients belonging to specific departments, which resulted in a rescreening strategy that was both low cost and effective. Further recognition of and adjustments in rescreening criteria remain paramount in different prevalence settings of COVID-19.

\section{Disclosure}

The authors report no conflicts of interest in this work.

\section{References}

1. World Health Organization. WHO Coronavirus (COVID-19) dashboard; 2021. Available from: https://covid19.who.int/. Accessed September 21, 2021.

2. Zhou Q, Gao Y, Wang X, et al. Nosocomial infections among patients with COVID-19, SARS and MERS: a rapid review and meta-analysis. Ann Trans Med. 2020;8(10):629. doi:10.21037/atm-20-3324

3. Du Q, Zhang D, Hu W, et al. Nosocomial infection of COVID-19: a new challenge for healthcare professionals. Int J Mol Med. 2021;47 (4):1. doi:10.3892/ijmm.2021.4864.

4. Lechien JR, Chiesa-Estomba CM, Place S, et al. Clinical and epidemiological characteristics of 1420 European patients with mild-tomoderate coronavirus disease 2019. J Intern Med. 2020;288(3):335344. doi:10.1111/joim.13089

5. Li R, Tian J, Yang F, et al. Clinical characteristics of 225 patients with COVID-19 in a tertiary Hospital near Wuhan, China. $J$ Clin Virol. 2020;127:104363. doi:10.1016/j.jcv.2020.104363

6. Wang $\mathrm{D}, \mathrm{Hu} \mathrm{B}, \mathrm{Hu} \mathrm{C}$, et al. Clinical characteristics of 138 hospitalized patients with 2019 Novel Coronavirus-infected pneumonia in Wuhan, China. JAMA. 2020;323(11):1061-1069. doi:10.1001/ jama.2020.1585

7. Popov GT, Baymakova M, Vaseva V, Kundurzhiev T, Mutafchiyski V. Clinical characteristics of hospitalized patients with COVID-19 in Sofia, Bulgaria. Vector Borne Zoonotic Dis. 2020;20(12):910-915. doi:10.1089/vbz.2020.2679

8. Zhang JJ, Dong X, Cao YY, et al. Clinical characteristics of 140 patients infected with SARS-CoV-2 in Wuhan, China. Allergy. 2020;75(7):1730-1741. doi:10.1111/all.14238

9. Ishii T, Kushimoto S, Katori Y, et al. Predictors of SARS-CoV-2 positivity based on RT-PCR Swab Tests at a drive-through outpatient clinic for COVID-19 screening in Japan. Tohoku J Exp Med. 2021;253(2):101-108. doi:10.1620/tjem.253.101

10. Huang T, Guo Y, Li S, et al. Application and effects of fever screening system in the prevention of nosocomial infection in the only designated hospital of coronavirus disease 2019 (COVID-19) in Shenzhen, China. Infect Control Hosp Epidemiol. 2020;41(8):978981. doi:10.1017/ice.2020.119

11. Anschau F, Worm PV, Kopittke L, et al. Smart Check - COVID-19 triage system: evaluation of the impact on the screening time and identification of clinical manifestations of SARS-CoV-2 infection in a public health service. Int J Clin Pract. 2021:e14610. doi:10.1111/ ijcp. 14610 
12. Stacevičienė I, Burokienė S, Steponavičienè A, Vaičiūnienė D, Jankauskienè A. A cross-sectional study of screening for coronavirus disease 2019 (COVID-19) at the pediatric emergency department in Vilnius during the first wave of the pandemic. Eur $J$ Pediatr. 2021;180(7):2137-2145. doi:10.1007/s00431-021-03999-Z

13. Soltan AAS, Kouchaki S, Zhu T, et al. Rapid triage for COVID-19 using routine clinical data for patients attending hospital: development and prospective validation of an artificial intelligence screening test. Lancet Digit Health. 2021;3(2):e78-e87. doi:10.1016/S2589-7500(20)30274-0

14. Peng L, Liu KY, Xue F, Miao YF, Tu PA, Zhou C. Improved early recognition of Coronavirus disease-2019 (COVID-19): single-center data from a Shanghai Screening Hospital. Arch Iran Med. 2020;23 (4):272-276.

15. Trahan MJ, Mitric C, Malhamé I, Abenhaim HA. Screening and testing pregnant patients for SARS-CoV-2: first-wave experience of a designated COVID-19 hospitalization centre in Montréal. J Obstet Gynaecol Can. 2021;43(5):571-575.

16. Kuzan TY, Murzoğlu Altıntoprak K, Çiftçi H, et al. A comparison of clinical, laboratory and chest $\mathrm{CT}$ findings of laboratory-confirmed and clinically diagnosed COVID-19 patients at first admission. Diagn Interv Radiol. 2021;27(3):336-343. doi:10.5152/dir.2020.20270

17. Stessel B, Callebaut I, Polus F, et al. Evaluation of a comprehensive pre-procedural screening protocol for COVID-19 in times of a high SARS CoV-2 prevalence: a prospective cross-sectional study. Ann Med. 2021;53(1):337-344. doi:10.1080/07853890.2021.1878272

18. Sharma S, Wazir S, Kumar RK. Universal screening for SARS-CoV2 in asymptomatic pregnant women: a multi-center experience. $J$ Obstet Gynaecol India. 2021;71(suppl 1):1-4.

19. Jung J, Kim J, Lim JS, Kim EO, Kim MN, Kim SH. Pitfall of universal pre-admission screening for SARS-CoV-2 in a low prevalence country. Viruses. 2021;13(5):804. doi:10.3390/v13050804

20. Salaouatchi MT, Mahadeb B, Clevenbergh P, et al. Efficacy of systematic coronavirus screening by PCR and viral cultures in addition to triage in limiting the spread of SARS-CoV-2 within a hemodialysis unit. J Nephrol. 2021:1-8. doi:10.1007/s40620-021-01115-w

21. Storey B, Bottomley M, Hammad S, et al. Regular RNA screening detects asymptomatic SARS-CoV-2 infection in haemodialysis patients. Nephrol Dial Transplant. 2021;36(6):1130-1132. doi:10.1093/ndt/gfab044

22. Al-Shamsi HO, Coomes EA, Alrawi S. Screening for COVID-19 in asymptomatic patients with cancer in a hospital in the United Arab Emirates. JAMA Oncol. 2020;6(10):1627-1628. doi:10.1001/ jamaoncol.2020.2548

23. Wang SM, Tao F, Hou Y, et al. Screening of SARS-CoV-2 in 299 hospitalized children with hemato-oncological diseases: a multicenter survey in Hubei, China. Curr Med Sci. 2020;40(4):642-645. doi:10.1007/s11596-020-2228-7

24. Fujiwara Y, Sato Y, Wang X, et al. Screening for COVID-19 in symptomatic cancer patients in a cancer hospital. Cancer Cell. 2020;38(5):609-610. doi:10.1016/j.ccell.2020.09.017

25. Chang E, Choi JS, Park TY, et al. A universal screening strategy for SARS-CoV-2 infection in intensive care units: Korean experience in a single hospital. Infect Chemother. 2020;52(3):352-359. doi:10.3947/ic.2020.52.3.352

26. Wee LE, Conceicao EP, Aung MK, et al. Rostered routine testing for healthcare workers and universal inpatient screening: the role of expanded hospital surveillance during an outbreak of COVID-19 in the surrounding community. Infect Control Hosp Epidemiol. 2021;19. doi:10.1017/ice.2021.366

27. Saidel-Odes L, Shafat T, Nativ R, Borer A, Nesher L. SARS-CoV-2 universal screening upon adult hospital admission in Southern Israel. J Hosp Infect. 2021;114:167-170. doi:10.1016/j.jhin.2021.04.026

28. Alsuhaibani MA, Kobayashi T, Trannel A, et al. Coronavirus disease 2019 (COVID-19) admission screening and assessment of infectiousness at an academic medical center in Iowa, 2020. Infect Control Hosp Epidemiol. 2021:1-5. doi:10.1017/ice.2021.294
29. Krüger S, Leskien M, Schuller P, et al. Performance and feasibility of universal PCR admission screening for SARS-CoV-2 in a German tertiary care hospital. J Med Virol. 2021;93(5):2890-2898. doi:10.1002/jmv.26770

30. Scheier T, Schibli A, Eich G, et al. Universal admission screening for SARS-CoV-2 infections among hospitalized patients, Switzerland, 2020. Emerg Infect Dis. 2021;27(2):404-410. doi:10.3201/ eid2702.202318

31. Uchida S, Uwamino Y, Uno S, et al. Universal polymerase chain reaction screening for severe Acute Respiratory Syndrome Coronavirus 2 in asymptomatic patients before hospital admission in Tokyo, Japan. J Clin Virol. 2021;142:104915. doi:10.1016/j. jcv.2021.104915

32. Stadler RN, Maurer L, Aguilar-Bultet L, et al. Systematic screening on admission for SARS-CoV-2 to detect asymptomatic infections. Antimicrob Resist Infect Control. 2021;10(1):44. doi:10.1186/s13756021-00912-z

33. Ayenigbara IO, Adeleke OR, Ayenigbara GO, Adegboro JS, Olofintuyi OO. COVID-19 (SARS-CoV-2) pandemic: fears, facts and preventive measures. Germs. 2020;10(4):218-228. doi:10.18683/germs.2020.1208

34. Arefi MF, Poursadeqiyan M. A review of studies on the COVID-19 epidemic crisis disease with a preventive approach. Work. 2020;66 (4):717-729.

35. Rosolanka R, Henao-Martinez AF, Pisney L, Franco-Paredes C, Krsak M. COVID-19: a review of current knowledge regarding exposure, quarantine, isolation and other preventive measures. Ther Adv Infect Dis. 2021;8:20499361211032039. doi:10.1177/ 20499361211032039

36. Abbas M, Robalo Nunes T, Martischang R, et al. Nosocomial transmission and outbreaks of coronavirus disease 2019: the need to protect both patients and healthcare workers. Antimicrob Resist Infect Control. 2021;10(1):7. doi:10.1186/s13756-020-00875-7

37. Kang SJ, Jung SI. Age-related morbidity and mortality among patients with COVID-19. Infect Chemother. 2020;52(2):154-164. doi:10.3947/ic.2020.52.2.154

38. Liu Z, Chu R, Gong L, Su B, Wu J. The assessment of transmission efficiency and latent infection period in asymptomatic carriers of SARS-CoV-2 infection. Int $J$ Infect Dis. 2020;99:325-327. doi:10.1016/j.ijid.2020.06.036

39. Gupta P, Muthukumar N, Rajshekhar V, et al. Neurosurgery and neurology practices during the Novel COVID-19 pandemic: a consensus statement from India. Neurol India. 2020;68(2):246-254. doi:10.4103/0028-3886.283130

40. Arimappamagan A, Vilanilam G, Pandey P. Is elective neurosurgery justified during COVID-19 pandemic? Neurol India. 2021;69(1):2125. doi:10.4103/0028-3886.310113

41. Tsermoulas G, Zisakis A, Flint G, Belli A. Challenges to neurosurgery during the Coronavirus disease 2019 (COVID-19) pandemic. World Neurosurg. 2020;139:519-525. doi:10.1016/j. wneu.2020.05.108

42. Agarwal N, Raheja A, Suri A. Guidelines for preoperative testing for neurosurgery in Coronavirus disease 2019 (COVID-19) era: Indian viewpoint amidst global practice. World Neurosurg. 2021;146:103112. doi:10.1016/j.wneu.2020.10.086

43. Shaheen S, Awwad O, Shokry K, et al. Rapid guide to the management of cardiac patients during the COVID-19 pandemic in Egypt: "a position statement of the Egyptian Society of Cardiology". Egypt Heart J. 2020;72(1):30.

44. Harky A, Chor CYT, Khare Y. COVID-19 and its impact on cardiology service. Acta Biomed. 2020;91(4). doi:10.1136/openhrt-2020001359

45. Sahu A, Kapoor A. Guiding principles for cardiology care in times of the COVID-19 pandemic: gazing through the crystal ball. Ann Card Anaesth. 2020;23(4):532-536. doi:10.4103/aca.ACA_150_20 
46. Yasmin F, Shujauddin SM, Naeem A, et al. Exploring the impact of the COVID-19 pandemic on provision of cardiology services: a scoping review. Rev Cardiovasc Med. 2021;22(1):83-95. doi:10.31083/j.rcm.2021.01.241

47. Anker SD, Butler J, Khan MS, et al. Conducting clinical trials in heart failure during (and after) the COVID-19 pandemic: an expert consensus position paper from the Heart Failure Association (HFA) of the European Society of Cardiology (ESC). Eur Heart J. 2020;41 (22):2109-2117. doi:10.1093/eurheartj/ehaa461

48. Zhang Y, Coats AJS, Zheng Z, et al. Management of heart failure patients with COVID-19: a joint position paper of the Chinese Heart Failure Association \& National Heart Failure Committee and the Heart Failure Association of the European Society of Cardiology. Eur J Heart Fail. 2020;22(6):941-956. doi:10.1002/ ejhf.1915
49. Lin KC, Wang CC, Huang WC, Hwang JJ. Considerations when managing heart failure during the COVID-19 pandemic-consensus from the Taiwan Society of Cardiology. Acta Cardiol Sin. 2021;37(2):125-129.

50. Garg N, McClafferty B, Ramgobin D, Golamari R, Jain R, Jain R. Cardiology and COVID-19: do we have sufficient information? Future Cardiol. 2021;17(4):705-711. doi:10.2217/fca-2020-0126

\section{Publish your work in this journal}

The Journal of Multidisciplinary Healthcare is an international, peerreviewed open-access journal that aims to represent and publish research in healthcare areas delivered by practitioners of different disciplines. This includes studies and reviews conducted by multidisciplinary teams as well as research which evaluates the results or conduct of such teams or healthcare processes in general. The journal covers a very wide range of areas and welcomes submissions from practitioners at all levels, from all over the world. The manuscript management system is completely online and includes a very quick and fair peer-review system. Visit http://www.dovepress.com/testimonials. php to read real quotes from published authors. 